\title{
Controlling the morphology of nanocrystal-polymer composites for solar cells
}

\author{
Dr. Wendy U. Huynh ${ }^{\ddagger}$, Dr. Janke J. Dittmer ${ }^{\ddagger}$, Mr. William C. Libby, Mr. Gregory L. \\ Whiting, Prof. A. Paul Alivisatos* \\ Department of Chemistry, University of California and Material Science Division, Lawrence \\ Berkeley National Laboratory, Berkeley CA94720, USA \\ Fax: 510-642-6911 \\ * Both authors contributed equally to this work \\ *To whom correspondence should be addressed: $\underline{\text { alivis@uclink4.berkelev.edu }}$
}

\begin{abstract}
We have shown recently that the use of high aspect ratio inorganic nanorods in conjunction with conjugated polymers is a route to obtaining efficient solar cells processed from solution. Here, we demonstrate that the use of binary solvent mixtures in which one of the components is a ligand for the nanocrystals is effective in controlling the dispersion of nanocrystals in a polymer. By varying the concentration of the solvent mixture, phase separation between the nanocrystal and polymer could be tuned from a micron scale down to nanometer scale. In addition, we can achieve nanocrystal surfaces that are free of surfactant through the use of weak binding ligands, which can be removed through heating. Combined, the control of film morphology together with surfactant removal result in nanorod-polymer blend photovoltaic cells with high external quantum efficiency of $59 \%$ under $0.1 \mathrm{~mW} / \mathrm{cm}^{2}$ illumination at $450 \mathrm{~nm}$.
\end{abstract}

Keywords: photovoltaic, nanorod, conjugated polymer, thermal annealing, binary solvent mixture 


\section{Introduction}

Solar cells assembled from blending inorganic nanorods with polymers have the potential of bridging the efficiency gap that exists between organic and inorganic photovoltaic materials. [1] Although advancements in polymer solar cell technology have resulted in plastic devices with solar power conversion efficiencies of $2.5 \%,{ }^{[2,3]}$ these devices have not attained the efficiencies of conventional solar cells that are typically above $10 \% .{ }^{[4]}$ One of the main factors limiting the performance of polymeric devices is the inefficient charge transport due to the low carrier mobilities as compared to bulk inorganic semiconductors. Shape control of nanocrystals from spherical to rod-like forms allows for efficient one-dimensional electrical transport, which, as seen in Si nanowires, can reach carrier mobilities comparable to bulk values. ${ }^{[5]}$ Additionally, the synthesis of nanorods in solution at temperatures below $300^{\circ} \mathrm{C}$ and subsequent re-dispersion of the particles in a variety of solvents ${ }^{[6,7]}$ permit the use of low cost techniques in the fabrication of large-area solar panels. Combining nanorods with a good hole-transporting polymer, such as poly(3-hexylthiophene) (P3HT) (Fig. 1a), enables the good transport characteristics of inorganic semiconductors to be used without sacrificing the solution processibility of organic materials. However, as the nanorods become longer, better 1-D transporters, and move towards the nanowire regime, it becomes increasingly difficult to solubilize them. Here, we discuss in detail how these difficulties can be overcome and in particular, how we control the morphology of nanorod-polymer blends. These findings lead to a highly efficient hybrid solar cell that we have reported elsewhere. ${ }^{[1]}$

Applications of these nanocomposites in solar cells rely on the ability to disperse the nanoparticles within the polymer in order to create a high interfacial surface area for charge 
transfer between the two materials. And, as in other optical applications, which use hybrid materials, such as colour emitters, ${ }^{[8]}$ light emitting diodes (LEDs), ${ }^{[9]}$ solar concentrators, ${ }^{[10]}$ effective dispersion of nanoparticles is necessary for optical clarity. The interaction of nanoparticles with polymers is mainly controlled by organic ligands adsorbed onto the surface of the nanoparticles, which make them soluble and passivate the surface for stability. In composite electronic devices, such as LEDs, photodetectors and solar cells, the presence of an organic ligand can severely reduce the device efficiency by impeding the transfer of charges between nanocrystal and polymer, and the transport of electrons between adjacent nanocrystals. ${ }^{[1]}$ However, to disperse polar nanorods with lengths of up to 100 nanometers (nm) at high concentration into a relatively non-polar polymer is a challenge, and it is difficult to do so such that the interface is free of organic surfactants. Here, we demonstrate that the use of binary solvent mixtures in which one of the components is a ligand for the nanocrystals is effective in controlling the dispersion of nanocrystals in a polymer. In addition, we can achieve nanocrystal surfaces that are free of surfactant through the use of weak binding ligands, which can be removed through heating. The use of the binary solvent mixture resulted in an increase in power conversion efficiency of more than $50 \%$. Further enhancement of the device performance through heat treatment enabled us to fabricate photovoltaic devices with external quantum efficiencies of up to $59 \%$ under $0.1 \mathrm{~mW} / \mathrm{cm}^{2}$ illumination at $450 \mathrm{~nm}$. 


\section{Results and Discussion}

\subsection{Morphology Control}

\subsubsection{Solvent Mixtures}

We used a solvent mixture in order to control phase separation in a film with a high concentration of nanocrystals (up to 90 weight \%) in P3HT down to the nanometer scale. The aim is to enhance the solubility of the nanocrystals by simultaneously using a good solvent and ligand for CdSe nanocrystals and a good solvent for the polymer for solution processing. The weak-binding Lewis base, pyridine, with its relatively low boiling point of $116^{\circ} \mathrm{C}$, was chosen as a ligand for the nanocrystals with the aim of facile removal. Pyridine treated nanocrystals of various shapes and sizes (Fig. 2) were co-dissolved with P3HT in a mixture of $4 \%$ to $12 \%$ by volume (vol. \%) pyridine in chloroform to create a uniform film consisting of dispersed particles in polymer when spin cast. The optimal amount of pyridine to cover the nanocrystal surface is determined by the number of non-passivated $\mathrm{Cd}$ surface sites on the nanoparticle. Pyridine is miscible in chloroform, so that there is a twofold solubility increase for the nanocrystals: (a) pyridine coated nanocrystals are more soluble in chloroform than their naked counterparts and (b) they are highly soluble in the excess pyridine that is not bound to nanocrystals. ${ }^{[12]}$ Too much pyridine, however, is to be avoided, as this mediates the precipitation of $\mathrm{P} 3 \mathrm{HT}$, which is very soluble in chloroform and insoluble in pyridine. Therefore, there are three regimes:

I. The low pyridine concentration regime: Insufficient solubility of the nanocrystals results in large-scale phase separation in the blend films promoted by nanocrystal flocculation.

II. The intermediate pyridine concentration regime: Provided that the polymer is still sufficiently soluble in a miscible blend of the two solvents, the solubility enhancement 
in the nanocrystal component of the blend solution will lead to intimate mixing of the two semiconductors and therefore prevent phase separation upon spin coating.

III. The high pyridine concentration regime: As pyridine is a non-solvent for the polymer component, we expect large-scale phase separation promoted by the flocculation of polymer chains.

To investigate the morphology of nanocrystal-polymer films, we can use surface sensitive techniques, such as atomic force microscopy (AFM), and bulk sensitive techniques such as transmission electron microscopy (TEM). An example of regime I is shown in Fig. 3 for a blend of 90 wt. $\% 7 \mathrm{~nm}$ by $7 \mathrm{~nm}$ nanocrystals in P3HT that was spun from a single solvent of chloroform. In this AFM image we can see phase separation on a scale of several microns, which could also be detected under an optical microscope and even with the bare eye as the film scattered light. Light scattering is undesirable in thin film photovoltaic cells, as it can decrease the fraction of light absorbed.

The study of the surfaces of nanocrystal-polymer blend films can be greatly enhanced by using AFM in the tapping mode (TM), as it is often possible to identify local differences in the composition of the film by comparing the phase and topography image. ${ }^{[13]}$ To illustrate the transition from regime I to regime II, Fig. 4 shows the AFM-TM topography and phase images for $5 \mu \mathrm{m}$ scan areas of $9 \mathrm{~nm}$ by $13 \mathrm{~nm}$ nanorod-P3HT blend films spun from solvent mixtures with low and intermediate pyridine concentrations. Whereas the topography of these films is very rough for low pyridine concentration, an intermediate concentration yields much smoother films. The corresponding AFM-TM phase images demonstrate that the surface roughness relates to phase separation. Phase separation between the nanocrystals and polymer do not yield single material domains and as such, it is not possible to identify the individual polymer and 
nanocrystal areas. At low pyridine concentration, there is clear evidence for local variations in the composition of the film, whereas at intermediate pyridine concentration the phase image is very smooth. We can therefore attribute these two concentrations to regime I and II, respectively. To express these results in a quantitative manner, we have determined the root mean square (RMS) of the film roughness from AFM topography images as a function of pyridine concentration (Fig. 5). The RMS roughness decreases by an order of magnitude, as the pyridine concentration increases from 0 to 5 vol. \%. Between 5 and 12 vol. \% pyridine concentrations, there is only a slight increase in the RMS roughness, whereas there is an order of magnitude increase, as the pyridine concentration is taken from 12 to $20 \mathrm{vol}$ \%. Using the above scheme we can attribute the concentration range from 0 to 5 vol. \% to regime I, 5 to 12 vol. \% to regime II and 12 to 20 vol. \% to regime III. These concentration values are for a fixed overall concentration of nanocrystals and polymer in the binary solution. For the $90 \mathrm{wt} . \%$ of CdSe nanocrystals in P3HT used in this study, the partial concentrations were $45 \mathrm{~g} / 1$ and $5 \mathrm{~g} / 1$ respectively.

\subsubsection{Device Performance}

Separation of charges only occurs for excitons that are created within the exciton diffusion range of a nanocrystal-polymer interface. ${ }^{[14]}$ As the single-material domain size decreases as a consequence of better nanocrystal dispersion, an increase in the external quantum efficiency (EQE) of a photovoltaic constructed from the blend is predicted (Fig. 1b). In photovoltaic devices based on a conjugated polymer/methanofullerene blend, effective dispersion of the methanofullerene can enhance the EQE by a factor of two over films with larger methanofullerene domains. ${ }^{[2,15]}$ The EQE, which is the percentage of electrons collected per incident photon, can be used as a measure of the efficiency of charge separation given that 
the following quantities are comparable for a set of devices: (i) incident light intensity, (ii) fraction of light absorbed, and (iii) charge collection efficiency at the electrodes, which is mainly given by the choice of electrodes. These three conditions are met for the devices for which EQE data are presented in Fig. 5. ${ }^{[16]}$ Fig. 5 shows the pyridine dependence of the maximum EQE under approximately $0.1 \mathrm{~mW} / \mathrm{cm}^{2}$ illumination for blends of $9 \mathrm{~nm}$ by 13 nanocrystals and P3HT. The $\mathrm{E}$ increases by a factor of 1.4 in going from regime I to regime II and then decreases again for regime III. The maximum efficiency of $35 \%$ is found for a pyridine concentration of 8 vol. \% in the solvent mixture. In comparing the pyridine dependence of the EQE with that of the RMS roughness we find an inverse dependence: the highest EQE is reached close to the lowest RMS film roughness, which corresponds to the smallest phase separation, whereas for high RMS film roughness the EQE is significantly reduced. The open-circuit voltage and fill factor for these devices do not change with pyridine concentration. This confirms the hypothesis that large-scale phase separation is detrimental for photovoltaic performance.

A similar dependence of the EQE on pyridine concentration in the binary solvent system exists for $7 \mathrm{~nm}$ spherical nanocrystals dispersed in P3HT. The maximum EQE is also at 8 vol. \% pyridine concentration, which is comparable to the value found for the low aspect ratio nanorods described above. For a fixed nanocrystal concentration, the optimal concentration of pyridine is determined by the surface-to-volume ratio of the nanocrystal. For devices comprised of $3 \mathrm{~nm}$ by $100 \mathrm{~nm}$ nanorods, the best devices are cast from solutions containing 12 vol. \% pyridine, whereas, devices with $7 \mathrm{~nm}$ by $60 \mathrm{~nm}$ nanorods require only 4 vol. \% pyridine. The $3 \mathrm{~nm}$ diameter nanorods have a factor of two higher surface-to-volume ratio than the $7 \mathrm{~nm}$ nanorods. More pyridine is required to maintain the surface of the thinner nanorods covered with pyridine, as these bound pyridine molecules are in dynamic equilibrium with free pyridine in solution. 


\subsection{Removal of surface ligands}

Heat treatment is an effective method to enhance the mobility of organic molecules bound to an inorganic surface and treatment of the nanocomposite near the glass transition temperature of the polymer enables the movement of these molecules within the film towards the surface. In blends, thermal annealing has been used to promote the equilibrium morphology of a spin cast film and in some cases to enhance phase separation and crystallization within the composite. ${ }^{[17]}$ Here, the elimination of the pyridine ligand with heat treatment is shown to be effective in improving the performance of the blend film in a photovoltaic device.

The normalized photocurrent measured for a $90 \mathrm{wt} . \% 3 \mathrm{~nm}$ by $60 \mathrm{~nm}$ CdSe nanorods in P3HT spin cast from a solvent of 10 vol. \% pyridine in chloroform (Fig. 6) is given in Fig. 7 a. The absolute maximum EQE is $15 \%$ under $0.1 \mathrm{~mW} / \mathrm{cm}^{2}$ illumination at $455 \mathrm{~nm}$ under flowing argon. Upon heating at $120^{\circ} \mathrm{C}$ under a reduced pressure of approximately $50 \mathrm{mTorr}$ for 3 hours and cooling for 8 hours to room temperature, the photocurrent of the same device is enhanced significantly (Fig. 7a). A ratio of the photocurrent for the heat treated device to that of the device prior to heating shows an overall enhancement by a factor of 2.5 and a particularly strong increase by a factor of greater than 4 near $700 \mathrm{~nm}$ and greater than 6 near $650 \mathrm{~nm}$ (Fig. 7b).

\subsubsection{Interfacial Pyridine}

To understand the origin of this red EQE enhancement peak at $700 \mathrm{~nm}$, a device with only $3 \mathrm{~nm}$ by $60 \mathrm{~nm}$ CdSe nanorods was fabricated and heated under the same conditions. An analysis of the photocurrent prior and subsequent to heat treatment shows that there is only an enhancement feature centered around $700 \mathrm{~nm}$. We can therefore attribute this red increase in the blend photocurrent spectrum to the nanorods. Because the onset of absorption in these CdSe nanorods 
is at approximately $650 \mathrm{~nm}$ (Fig. 7b, inset), the presence of enhanced photocurrent at $700 \mathrm{~nm}$ in the device upon heating suggests that the effective diameter of the particles has increased. A red shift in the absorption of nanocrystals has been observed in superlattices relative to independent particles as a consequence of the coherent interaction of nanocrystals in close proximity in the absence of surface ligands. ${ }^{[18]}$ Moreover, it is possible for nanocrystals to be sintered together at relatively low temperatures compared to the bulk melting point ${ }^{[19]}$ to produce larger crystals. Consequently, heat treatment is seen to aid both in the removal of interfacial pyridine and in bringing nanorods closer together (Fig. 9a). This aggregation of neighbouring nanorods is likely to improve electron transport between nanorods, which occurs through thermally activated hopping. ${ }^{[20]}$ Furthermore, the removal of interfacial pyridine can also have the effect of enhancing charge transfer between CdSe and P3HT by bringing these two materials into closer electronic contact. These two effects most likely resulted in the overall photocurrent enhancement by a factor of about 2.5 across all absorbed wavelengths.

\subsubsection{Excess Pyridine}

The greatest photocurrent increase occurs in the region between $500 \mathrm{~nm}$ and $700 \mathrm{~nm}$, where an increase by a factor of greater than 6 is obtained for the $90 \mathrm{wt}$ \% CdSe blend device. In this wavelength region both CdSe and P3HT contribute significantly to the absorption of light. The relative contribution of each material component to the absorption spectrum can be compared with their contribution to the photocurrent spectrum. The absorption spectra (in units of optical density) of a series of devices with varying CdSe concentration can be decomposed into a linear combination of the individual CdSe and P3HT spectra (Fig. 8a). ${ }^{[21]}$ Likewise, the photocurrent spectra of the blend devices can be expressed as a combination of the CdSe photocurrent spectrum, given as $-\log (1-E Q E / 100)$ and the absorption spectrum of $\mathrm{P} 3 \mathrm{HT} .^{[22]}$ There is no 
change in the absorption between $400 \mathrm{~nm}$ and $700 \mathrm{~nm}$ of the blend device as a result of heating. For concentrations greater than 20 wt. \% CdSe in P3HT, the contribution of P3HT to the photocurrent is significantly less than the proportion of light that is absorbed by the polymer. In the 90 wt. \% CdSe device, the P3HT component is responsible for $60 \%$ of the absorption spectrum but the polymer only contributes to $42 \%$ of the photocurrent spectrum, given as $-\log (1-$ $E Q E / 100)$. This suggests that a substantial amount of light absorbed by the P3HT does not result in the generation of current and is lost either to non-radiative or radiative recombination pathways. However, upon heat treatment of these devices at $120^{\circ} \mathrm{C}$, the change in the photocurrent spectra yields $\mathrm{P} 3 \mathrm{HT}$ contributions that are closer to that in the absorption spectra of the device. For the heated 90 wt. \% CdSe device, the P3HT portion of the photocurrent spectrum rises to $63 \%$, comparable to its contribution to the absorption spectrum. An overall amplification of the $\mathrm{EQE}$ is observed from $60^{\circ} \mathrm{C}$ to $160^{\circ} \mathrm{C}$ heating temperature, decreasing once again at $180^{\circ} \mathrm{C}$ as aluminum migrates through the film and the device degrades (Fig. 8b). ${ }^{[23]}$ Correspondingly, the photoluminescence (PL) efficiency of a $60 \mathrm{wt}$ \% CdSe blend film as a function of treatment temperature, rises up to $120^{\circ} \mathrm{C}$, decreases thereafter and remains constant at higher temperature (Fig. 8c). Since the PL efficiency of CdSe in the blend is less than $0.1 \%$, the PL of the sample arises predominantly from $\mathrm{P} 3 \mathrm{HT}$. Heating of $\mathrm{P} 3 \mathrm{HT}$ is known to result in enhanced crystallinity, which quenches the PL efficiency. ${ }^{[24]}$ This effect has been observed in heated films of P3HT at as low a temperature as $40^{\circ} \mathrm{C} .{ }^{[25]}$ Increased crystallinity, therefore, explains the slight decrease in PL efficiency observed above $120^{\circ} \mathrm{C}$, but fails to account for the substantial increase in PL efficiency below $120^{\circ} \mathrm{C}$. Under annealing at lower temperatures, the removal of excess pyridine within the polymer is the likely cause for the increase in P3HT photoluminescence efficiency with increasing treatment temperature (Fig. 9b). This is because some of the photons absorbed in 
P3HT undergo non-radiative recombination at pyridine sites within the polymer in the untreated film and do not contribute to PL. After heat treatment, these photons can contribute to both radiative decay and charge transfer. Consequently, the removal of excess pyridine results in a larger $\mathrm{P} 3 \mathrm{HT}$ contribution to the photocurrent leading to the enhancement in EQE observed in the region between $500 \mathrm{~nm}$ and $700 \mathrm{~nm}$, where P3HT absorbs strongly.

\subsubsection{Heating and Device Performance}

Thermal treatment is especially important to enhance EQEs in high aspect ratio nanorod photovoltaic devices, which have a high surface-to-volume ratio and require higher concentrations of pyridine ( $>8$ vol. $\%$ ) in the spin casting solution. In devices consisting of these nanorods, there are large nanorod-nanorod, and nanorod-polymer interfacial areas containing pyridine as well as substantial amounts of excess pyridine. Removal of this pyridine resulted in the large EQE improvements of up to a factor of six observed in Fig. 7b. In contrast, nanorods of dimension $7 \mathrm{~nm}$ by $60 \mathrm{~nm}$ are blended with P3HT in solvents requiring only 4 vol. \% pyridine and the maximum EQE increase after heating is by only a factor of 1.3 (Fig. 10). In this case of nanorods with low surface-to-volume ratios, pyridine removal from thin films $(<200 \mathrm{~nm})$ results from merely pumping on the sample at low pressure $\left(<10^{-6} \mathrm{mbar}\right)$ and no improvement in performance is observed upon heat treatment. Moreover, heat treatment of thin films is detrimental to the open-circuit and fill factor as aluminum diffuses through a significant portion of the device. However, as the film thickness increases, thermal treatment becomes more important for removing pyridine from the device. At $210 \mathrm{~nm}$ thickness, the EQE increases upon heating by a maximum factor of 1.1 relative to the pristine devices. However, devices with 271 $\mathrm{nm}$ and $346 \mathrm{~nm}$ in thickness exhibit enhancements in the photocurrent of up to a factor of two and three respectively (Fig. 11). In future studies of photovoltaic devices using longer rods and 
nanorods aligned perpendicular to the substrate, improved electron transport would allow the use of thicker films to absorb more sunlight. In these thick films, heat treatment is expected to be necessary to remove pyridine and thus realize high performance devices.

Optimization of the morphology of nanocrystal-polymer blends with solvent mixtures and thermal treatment to remove interfacial and excess pyridine has yielded high efficiency photovoltaic devices. A device fabricated from $90 \mathrm{wt} \% 7 \mathrm{~nm}$ by $60 \mathrm{~nm}$ nanorods in P3HT spin cast from a 4 vol. \% pyridine in chloroform solvent mixture and thermally treated at $120^{\circ} \mathrm{C}$ achieves a maximum EQE of 59\% under $450 \mathrm{~nm}$ wavelength illumination at an intensity of approximately $0.1 \mathrm{~mW} / \mathrm{cm}^{2}$ (Fig. 10). This EQE is one of the highest EQE observed in polymer blend photovoltaic device.

\section{Conclusions}

In conclusion, we have shown that the performance of photovoltaic cells made of nanocomposites of inorganic nanocrystals and a conjugated polymer can attain external quantum efficiencies of up to $59 \%$. This high efficiency was a result of effectively dispersing nanorods in P3HT with an optimized pyridine-chloroform solvent mixture and subsequent removal of the ligand pyridine through thermal treatment. Dispersion of nanorods in the polymer is important for the creation of a large interfacial area for the transfer of charges between the two active materials. The strong enhancement of the photocurrent after heat treatment is explained by the removal of both interfacial and excess pyridine, which acts as a non-radiative recombination site

for excitons in the polymer. Furthermore, the removal of interfacial pyridine is likely to improve the photovoltaic conversion of excitons created in both materials, as the presence of interfacial surfactants is known to hinder the transfer of charges between the two components. In summary, 
we have shown that solvent mixtures and thermal annealing are important tools for processing hybrid inorganic-organic composites for electronic applications, because they can be used to produce dispersed nanorods relatively free of organic surfactants, necessary for processing, which otherwise would severely limit device efficiency.

\section{Experimental}

Nanocrystals were synthesized using pyrolysis of organometallic precursors in a mixture consisting mainly of trioctylphosphine oxide (TOPO) and tributyl- or trioctylphosphine and small amounts of various phosphonic acids. ${ }^{[6,26]}$ The recovered product was dispersed and washed three times in methanol to remove excess surfactant. Pyridine treatment of the nanocrystals to remove the surfactant used in the synthesis of nanorods was accomplished by dissolving the particles in pyridine and subsequent precipitation in hexanes. Whereas TOPO coated CdSe nanocrystals are soluble in hexanes, pyridine-coated particles are insoluble in hexanes. Repeating the pyridine treatment two to three times can effectively replace more than $95 \%$ of the TOPO on the nanocrystal surface with pyridine. ${ }^{[27]}$ The nanocrystals were characterized with transmission electron microscopy using a FEI Tecnai 12 120kV microscope. Thin films of CdSe-P3HT (regioregular P3HT from Aldrich) blends approximately 50-100 nm thick were investigated using TEM by casting a film on a $\mathrm{NaCl}$ IR window, floating the film in water and picking it up with a copper TEM grid. The morphology of the blend films was also characterized directly on devices via atomic force microscopy in tapping mode using a Nanoscope IIIa from Digital Instruments. Film thicknesses were determined via AFM.

Photovoltaic devices were fabricated from spin casting a solution of CdSe nanocrystals and P3HT in a pyridine-chloroform solvent mixture onto an ITO coated glass substrate under 
inert atmosphere, pumping for 12 hours under $<10^{-6}$ mbar and evaporating aluminum on top to obtain the structure depicted in Fig. 1b. The absorption of the CdSe-P3HT blend films was determined with an Agilent Chemstation UV/Vis spectrophotometer. Photocurrent measurements were completed using a 250 Watt tungsten light source coupled to an Acton SP150 monochromator as an illumination source and a Keithley 236 Source Measure Unit to obtain current and voltage. The light intensity was measured with a calibrated Graseby silicon photodiode.

Photoluminescence quenching experiments were completed on CdSe-P3HT films of thickness 100-200 nm spin cast on glass substrates. The absolute photoluminescence of the sample under excitation at $514 \mathrm{~nm}$ from an argon ion laser was measured with an integrating sphere following the method described by deMello et al. ${ }^{[28]}$

\section{Acknowledgements}

Supported by the National Renewable Energy Laboratory, grant XAD-9-18668-02 and the Department of Energy contract DE--AC03-76SF00098. We are grateful to the Robert D. Ogg Electron Microscopy Laboratory at the University of California, Berkeley for assistance with the TEM work. The authors also would like to thank L. Li for providing the $3 \mathrm{~nm}$ by $100 \mathrm{~nm} \mathrm{CdSe}$ nanorods and D. Milliron for valuable discussions. WUH thanks the Natural Sciences and Engineering Research Council of Canada for a fellowship. 


\section{Figure Captions}

Figure 1. a) Regioregular poly(3-hexylthiophene). b) Schematic of the structure of the nanorodpolymer blend photovoltaic device.

Figure 2 Low resolution TEM image of a) $7 \mathrm{~nm}$ by $7 \mathrm{~nm}$, b) $8 \mathrm{~nm}$ by $13 \mathrm{~nm}$, c) $3 \mathrm{~nm}$ by $60 \mathrm{~nm}$, and d) $7 \mathrm{~nm}$ by $60 \mathrm{~nm}$ CdSe nanocrystals.

Figure 3 AFM-TM topography image of a film consisting of 90 wt. $\% 7 \mathrm{~nm}$ by $7 \mathrm{~nm} \mathrm{CdSe}$ nanocrystals dispersed in P3HT, spin cast from chloroform. The scan area is $5 \mu \mathrm{m}$.

Figure 4 AFM-TM a) topography and b) phase images of films consisting of $90 \mathrm{wt} \% 8 \mathrm{~nm}$ by $13 \mathrm{~nm}$ CdSe nanocrystals dispersed in P3HT spin cast from 1 vol. \% and 8 vol. \% pyridine in chloroform. Images are presented at the same scale for a scan area of $5 \mu \mathrm{m}$.

Figure 5 Surface roughness (open circles) of films consisting of 90 wt. $\% 8 \mathrm{~nm}$ by $13 \mathrm{~nm} \mathrm{CdSe}$ nanocrystals dispersed in P3HT spin cast from various concentrations of pyridine in chloroform. The maximum EQE (solid diamonds) is shown of devices made from these films. Lines serve as a guide to the eye.

Figure 6 TEM image of a film consisting of $40 \mathrm{wt} . \% 3 \mathrm{~nm}$ by $60 \mathrm{~nm}$ CdSe nanorods dispersed in P3HT spin cast from a solvent consisting of 10 vol. \% pyridine in chloroform. 
Figure 7. a) Normalized photocurrent spectra for a $90 \mathrm{wt} \% 3 \mathrm{~nm}$ by $60 \mathrm{~nm}$ CdSe nanorods in P3HT device (open circles) and after annealing at $120^{\circ} \mathrm{C}$ (solid squares). b) Ratio of the EQE before and after heat treatment as a function of wavelength for $90 \mathrm{wt} . \% 3 \mathrm{~nm}$ by $60 \mathrm{~nm} \mathrm{CdSe}$ nanorods in P3HT device and a nanorod-only device. The inset shows the individual 1transmission spectra for $3 \mathrm{~nm}$ by $60 \mathrm{~nm}$ CdSe and P3HT.

Figure 8 a) Relative contribution of P3HT to the absorption (solid diamond, dashed line), photocurrent (open circle, solid line) and photocurrent after $120^{\circ} \mathrm{C}$ heat treatment (solid square, dashed line) of series of $3 \mathrm{~nm}$ by $60 \mathrm{~nm}$ nanorod devices in P3HT at various nanorod concentrations. b) The EQE of 90 wt. $\% 8 \mathrm{~nm}$ by $13 \mathrm{~nm} \mathrm{CdSe} \mathrm{in} \mathrm{P3HT} \mathrm{under} \sim 0.1 \mathrm{~mW} / \mathrm{cm}^{2}$ illumination at $515 \mathrm{~nm}$. The inset shows the PL efficiency of $60 \mathrm{wt} . \% 8 \mathrm{~nm}$ by $13 \mathrm{~nm}$ CdSe in P3HT samples under $514 \mathrm{~nm}$ excitation after heat treatment at various temperatures.

Figure 9. a) Removal of interfacial pyridine after heating. b) Removal of excess pyridne from P3HT after heating.

Figure 10 a) The EQE spectra of a 90 wt. \% $7 \mathrm{~nm}$ by $60 \mathrm{~nm}$ CdSe nanorods in P3HT (open circles) and after $120^{\circ} \mathrm{C}$ heat treatment (solid squares). b)Tthe corresponding current-voltage characteristics, under illumination of $0.1 \mathrm{~mW} / \mathrm{cm}^{2}$ at $515 \mathrm{~nm}$, for this device, which includes an open circuit voltage of $0.4 \mathrm{~V}$ and a fill factor of 0.5 .

Figure 11 The EQE spectra of devices from 90 wt. \% $7 \mathrm{~nm}$ by $60 \mathrm{~nm}$ CdSe nanorods in P3HT with thickness $212 \mathrm{~nm}, 271 \mathrm{~nm}$ and $346 \mathrm{~nm}$ a) before and b) after $120{ }^{\circ} \mathrm{C}$ heat treatment. 


\section{References and Notes}

[1] W. U. Huynh, J. J. Dittmer, A. P. Alivisatos, Science 2002, 295, 2427.

[2] S. E. Shaheen, C. J. Brabec, N. S. Sariciftci, F. Padinger, T. Fromherz, J. C. Hummelen, Appl. Phys. Lett. 2001, 78, 841.

[3] G. Yu, J. Gao, J. C. Hummelen, F. Wudl, A. J. Heeger, Science 1995, 270, 1789.

[4] M. A. Green, K. Emery, D. L. King, S. Igari, W. Warta, Prog. Photovoltaics 2001, 9 , 287.

[5] Y. Cui, X. F. Duan, J. T. Hu, C. M. Lieber, J. Phys. Chem. B 2000, 104, 5213.

[6] X. G. Peng, L. Manna, W. D. Yang, J. Wickham, E. Scher, A. Kadavanich, A. P. Alivisatos, Nature 2000, 404, 59.

[7] Z. A. Peng, X. G. Peng, J. Am. Chem. Soc. 2001, 123, 183.

[8] J. Lee, V. C. Sundar, J. R. Heine, M. G. Bawendi, K. F. Jensen, Adv. Mater. 2000, 12, 1102.

[9] B. O. Dabbousi, M. G. Bawendi, O. Onitsuka, M. F. Rubner, Appl. Phys. Lett. 1995, 66, 1316.

[10] K. Barnham, J. L. Marques, J. Hassard, P. O'Brien, Appl. Phys. Lett. 2000, 76, 1197.

[11] N. C. Greenham, X. G. Peng, A. P. Alivisatos, Phys. Rev. B. 1996, 54, 17628.

[12] H. Mattoussi, A. W. Cumming, C. B. Murray, M. G. Bawendi, R. Ober, Phys. Rev. B 1998, 58,7850 .

[13] S. N. Magonov, J. Cleveland, V. Elings, D. Denley, M.-H. Whangbo, Surf. Sci. 1997, 389, 201.

[14] D. S. Ginger, N. C. Greenham, Phys. Rev. B 1999, 59, 10622.

[15] L. S. Roman, M. R. Andersson, T. Yohannes, O. Inganas, Adv. Mater. 1997, 9, 1164. 
[16] The electrodes were chosen such that there is no barrier to charge collection. The shape of the corresponding I-V characteristics for this set of devices do not depend on the pyridine concentration, a further indication of the independence of charge collection on the morphology.

[17] J. J. Dittmer, E. A. Marseglia, R. H. Friend, Adv. Mater. 2000, 12, 1270.

[18] C. B. Murray, C. R. Kagan, M. G. Bawendi, Science 1995, 270, 1335.

[19] a) Y. A. Vlasov, N. Yao, D. J. Norris, Adv. Mater. 1999, 11, 165. b) B. A. Ridley, B. Nivi, J. M. Jacobson, Science 1999, 286, 746.

[20] D. S. Ginger, N. C. Greenham, J. Appl. Phys. 2000, 87, 1361.

[21] The absorption spectra, in units of optical density (log $\left.I_{0} / l\right)$, was modelled effectively as linear combination of the absoption spectra for films of CdSe and films of $\mathrm{P} 3 \mathrm{HT}$. By using this description of the absorption spectrum, we assume that the domains of P3HT and CdSe stack parallel to the substrate. This model of the absorption profile is in agreement with the distribution of CdSe and P3HT domains across the film thickness observed in TEM images of the film crosssection.

[22] The photocurrent spectrum, in units of -log [1-EQE/100], was modelled effectively as a linear combination of the photocurrent spectrum of CdSe, in the same units, and absoption spectrum or photocurrent spectrum for films of P3HT. It is necessary to the use the absorption spectra of P3HT for films with $>60$ wt. $\%$ CdSe because the photocurrent spectrum is antibatic in devices made of only $\mathrm{P} 3 \mathrm{HT}$. At concentrations > 60wt. \% the $\mathrm{P} 3 \mathrm{HT}$ response in the blend photocurrent spectrum is symbatic. 
[23] F. J. Esselink, G. Hadziioannou, Synth. Met. 1995, 75, 209.

[24] a) B. Xu, J. Lowe, S. Holdcroft, Thin Solid Films 1994, 243, 638. b) J. Cornil, D. A. dosSantos, X. Crispin, R. Silbey, J. L. Brédas, J. Am. Chem. Soc. 1998, 120, 1289.

[25] Y. Zhao, G. X. Yuan, P. Roche, M. Leclerc, Polymer 1995, 36, 2211.

[26] a) X. G. Peng, J. Wickham, A. P. Alivisatos, J. Am. Chem. Soc. 1998, 120, 5343. b) Z. A. Peng, X. G. Peng, J. Am. Chem. Soc. 2001, 123, 1389.

[27] M. Kuno, J. K. Lee, B. O. Dabbousi, F. V. Mikulec, M. G. Bawendi, J. Chem. Phys. 1997, 106, 9869.

[28] J. C. deMello, H. F. Wittmann, R. H. Friend, Adv. Mater. 1997, 9, 230. 


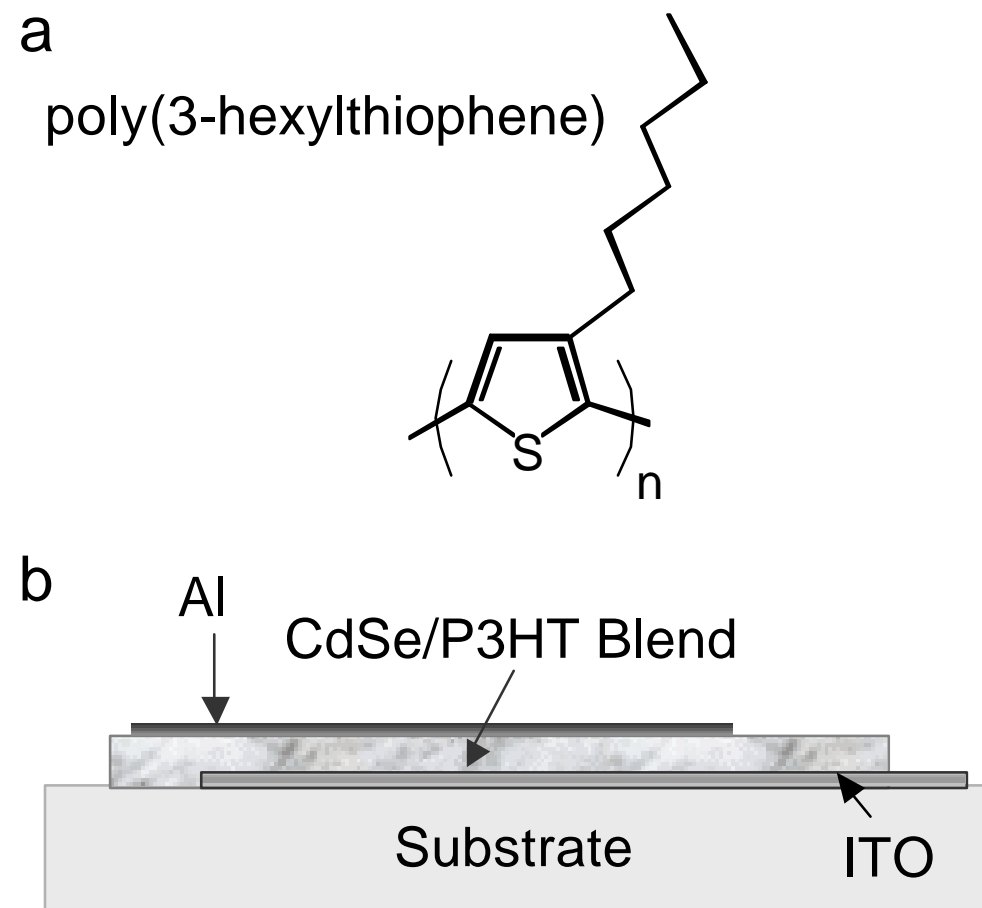

Figure 1

\section{Huynh et al.}




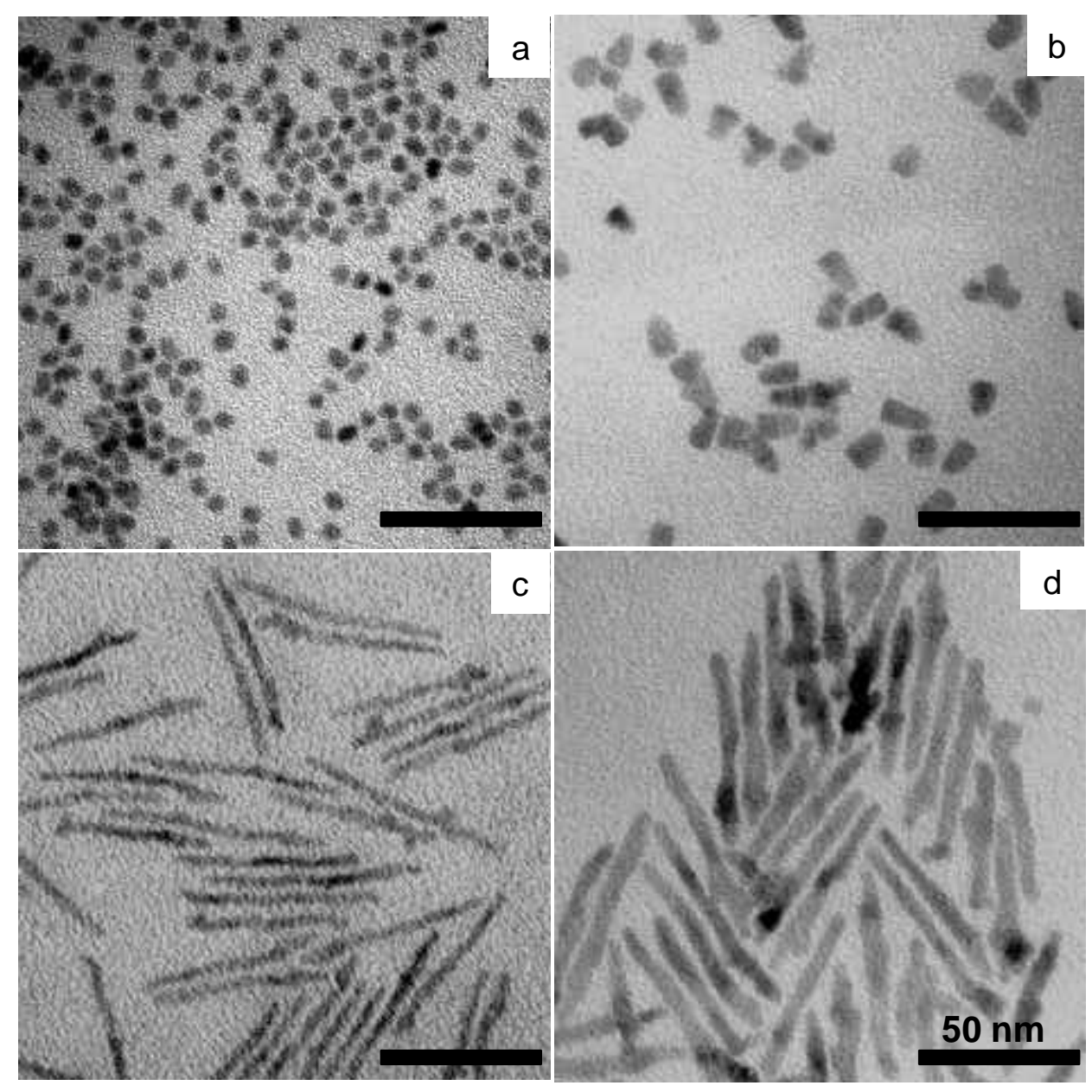

Figure 2

\section{Huynh et al.}






Figure 3

Huynh et al. 



Figure 4

\section{Huynh et al.}




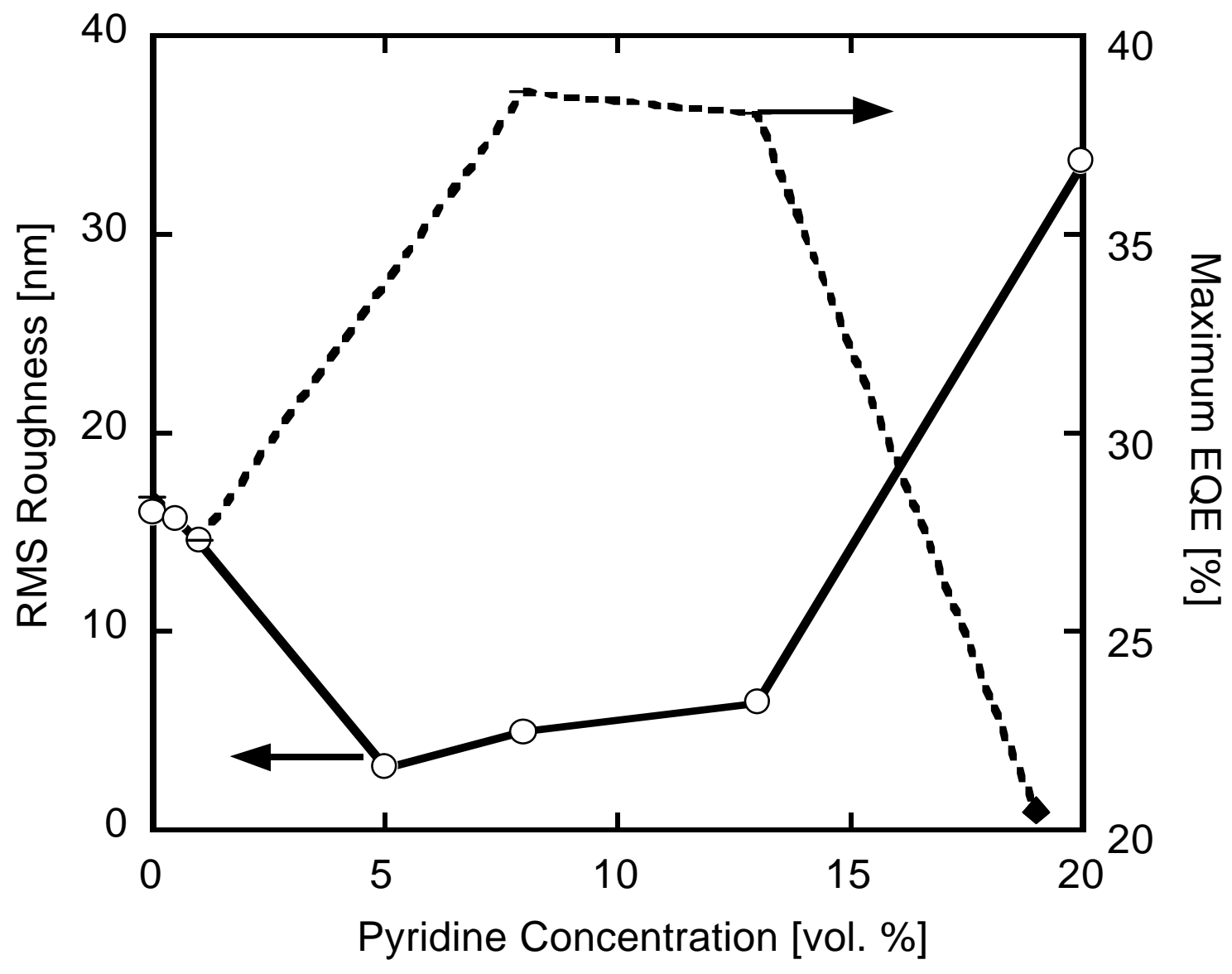

Figure 5

Huynh et al. 


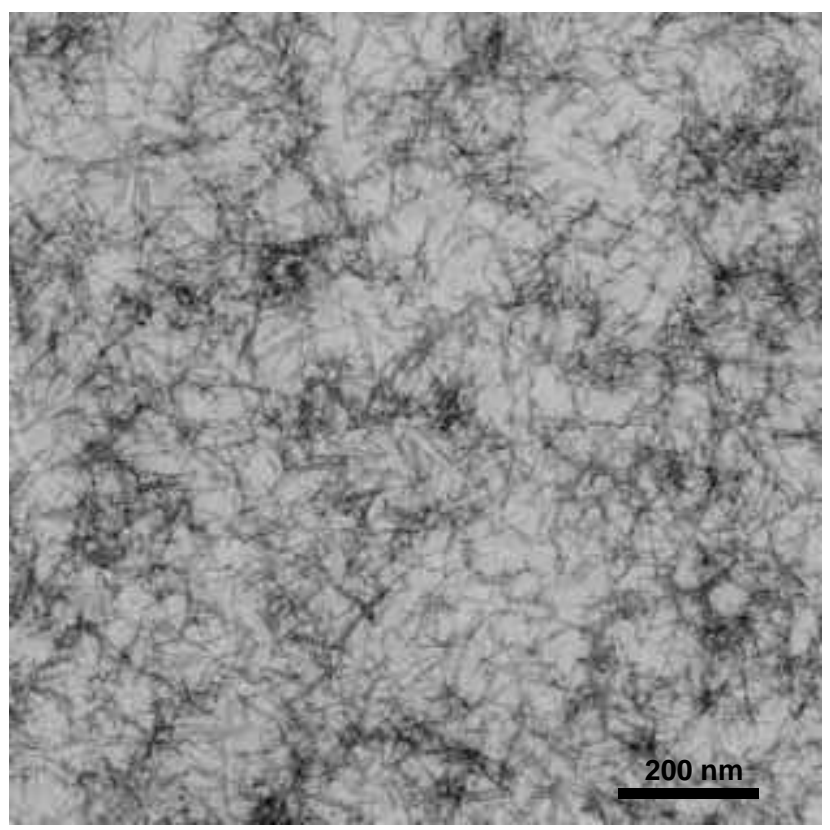

Figure 6

Huynh et al. 


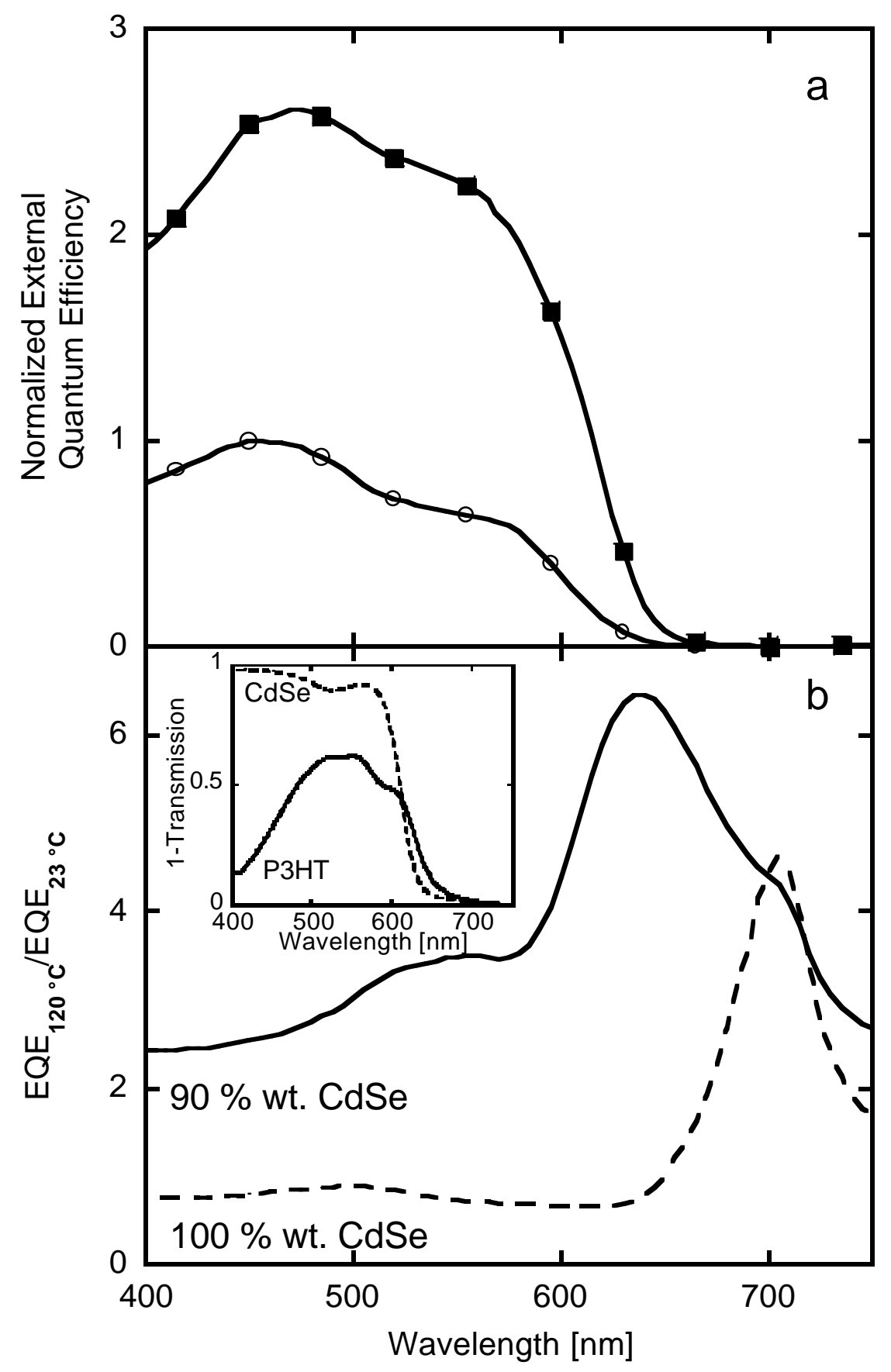

Figure 7

Huynh et al. 

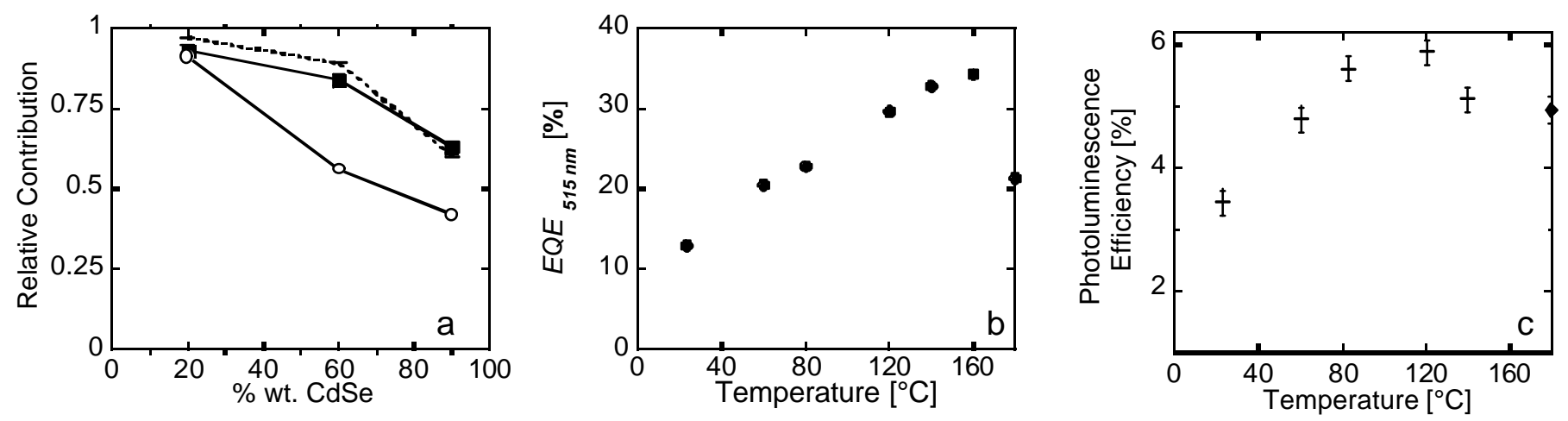

Figure 8

Huynh et al. 
a
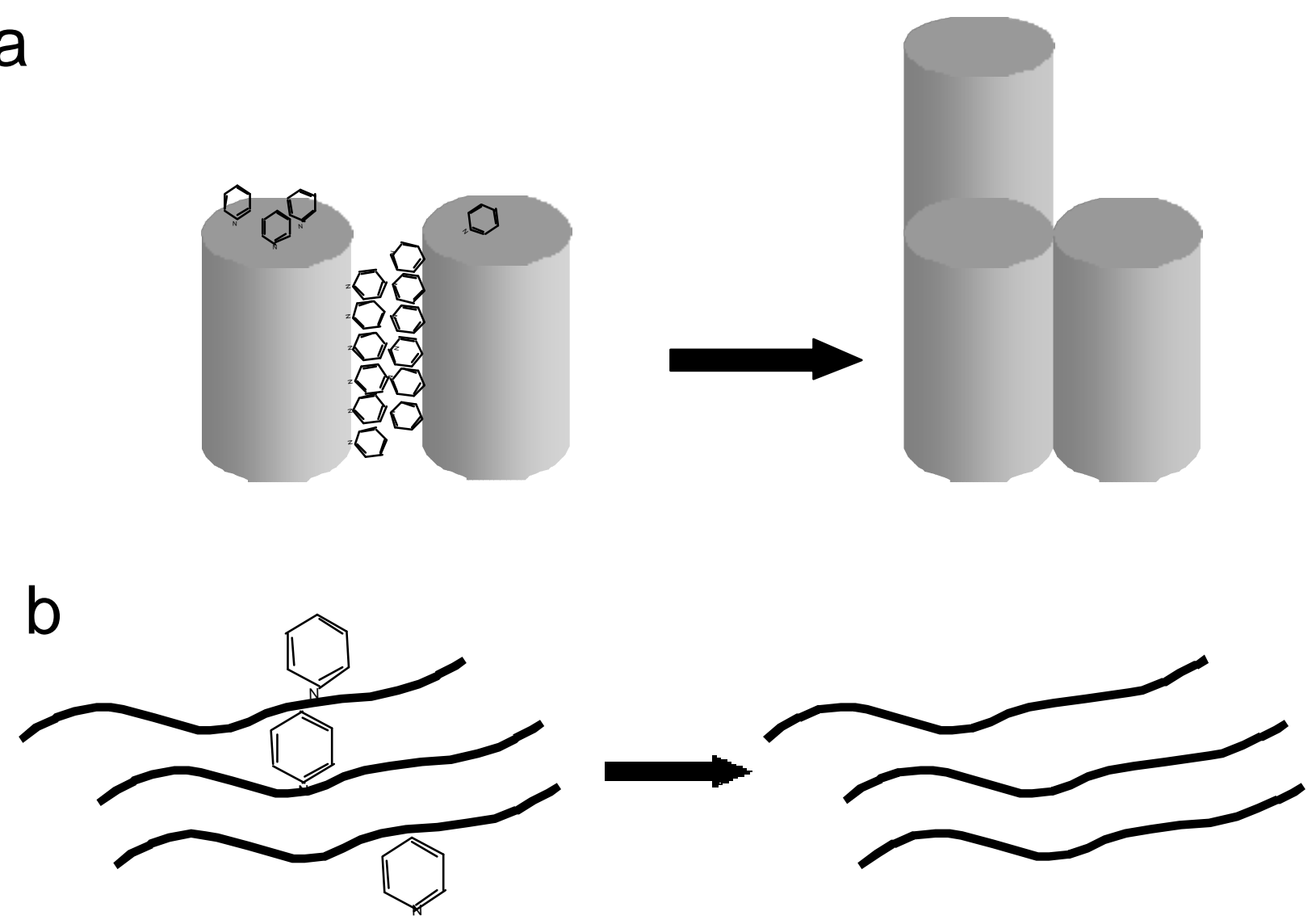

Figure 9

Huynh et al. 

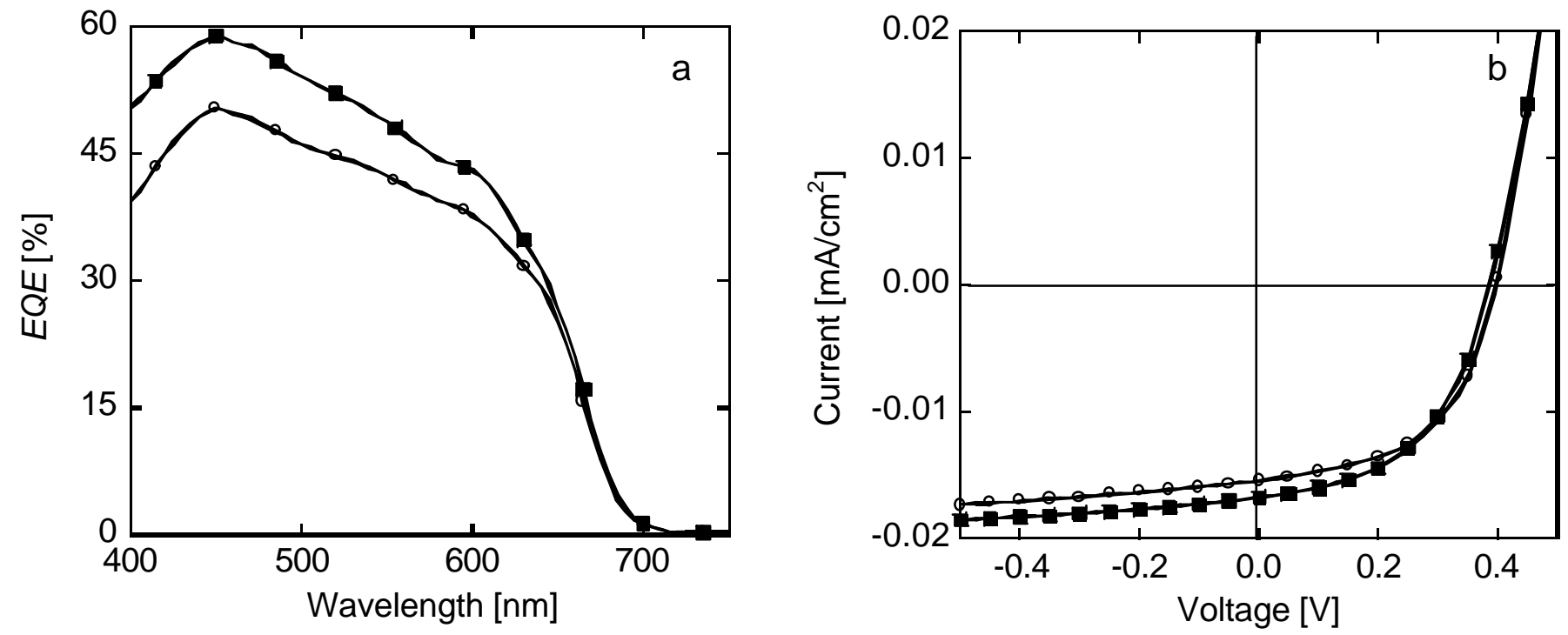

Figure 10

Huynh et al. 

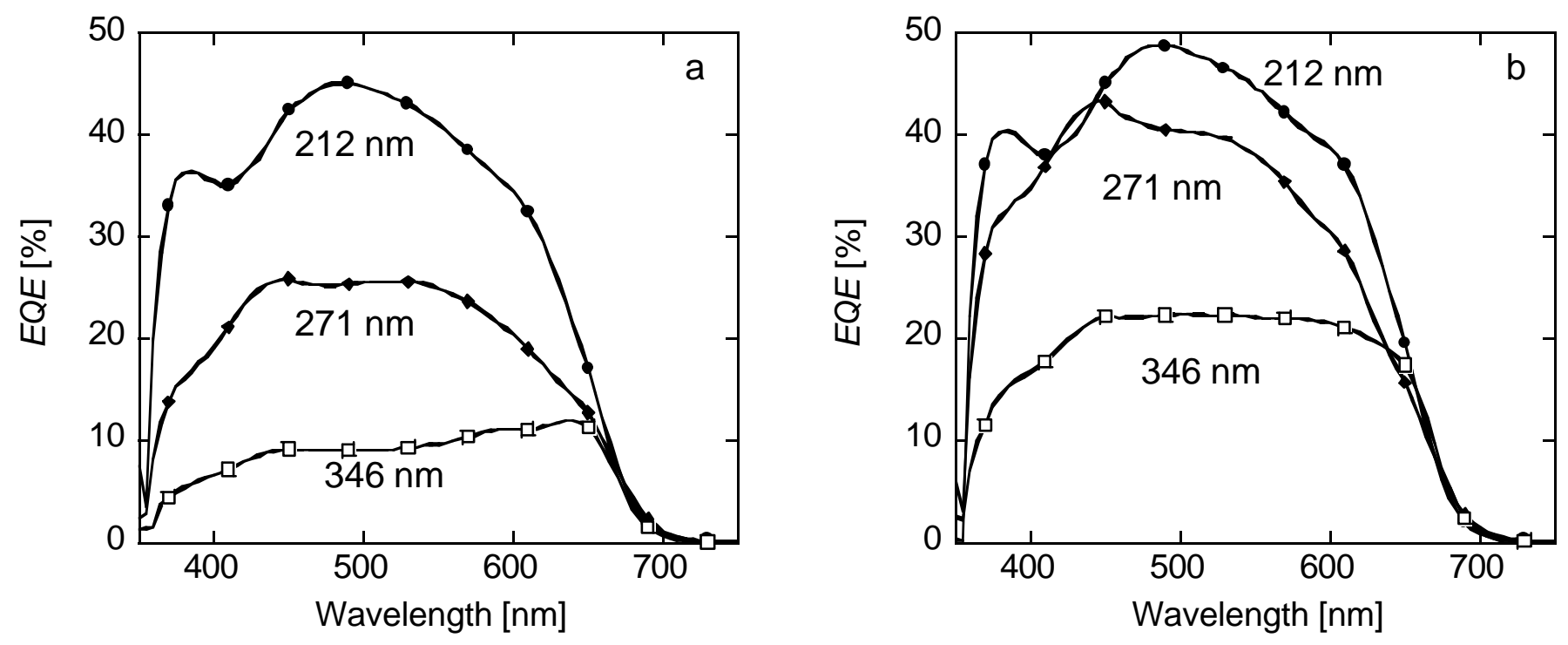

Figure 11

Huynh et al. 